\title{
ASSESSMENT OF REINFORCED CONCRETE STRUCTURES WITH DISTRIBUTED FIBRE OPTIC SENSORS
}

\author{
A. R. Brault ${ }^{*}$ and N. A. Hoult ${ }^{2}$ \\ ${ }^{1}$ Department of Engineering, University of Cambridge, Cambridge, United Kingdom. \\ ${ }^{2}$ Department of Civil Engineering, Queen's University, Kingston, Canada. \\ * Corresponding author
}

\begin{abstract}
The amount of concrete infrastructure expected to be built in the coming years will have significant adverse effects on the environment. The structural engineering community can help reduce this impact through efficient material use in design. In order to do this, comprehensive data sets that can be used to accurately assess the performance of reinforced concrete (RC) structures are required to ensure that optimisation comes without compromising public safety. Thus, the development of novel sensors that provide this comprehensive data is critical. Distributed fibre optic sensors have the potential to do this in a manner that is practical to implement, enabling detailed assessments of RC behaviour on site and in the lab. This paper demonstrates the capabilities of pairing commercially available fibre optic cables and a Rayleigh backscatter analysing unit to capture RC behaviour using distributed strain data. A series of beam tests were performed in the lab and a live load test was performed on a beam in a newly constructed building. The fibre optic sensors were found to be capable of measuring full distributed reinforcement strain profiles, full deflected shapes, and crack widths. However, the sensing techniques were unable to capture postyielding strain or deflection behaviour, and the accuracy of the deflection and crack width measurements decreased when cracks in the concrete surface exceeded $0.15 \mathrm{~mm}$ in width. The field monitoring data from a live load test demonstrated that externally bonded fibre optic sensors can provide information regarding support conditions, moment distribution, and cracking behaviour of building elements.
\end{abstract}

\section{Introduction}

The amount of reinforced concrete (RC) infrastructure that will be built in the coming years is significant, as it is expected that global cities will absorb an additional 2.5 billion people (UN Department of Economics and Social Affairs, 2018). This increase in concrete construction will have a significant negative impact on the environment since the production of cement alone accounts for approximately $8 \%$ of global carbon dioxide $\left(\mathrm{CO}_{2}\right)$ emissions (Andrew, 2018).

In light of this, it is important that any inefficiencies in RC design are reduced moving forward. Studies suggest that material savings of between $40 \%$ (Orr, 2012) and 70\% (Liew, 2017) are achievable in the design of concrete structures, though implementing efficient material use in practise is difficult for many reasons. One of these being that the industry must be certain that the removal of overly conservative assumptions in the design process will not compromise the structures' integrity, and in particular, safety. Understanding an RC element's response to loading in detail is therefore critical.

One method of providing structural engineers with the confidence to avoid over specifying material quantities in structures is by using monitoring data that comprehensively captures their behaviour. If detailed and distributed data was regularly captured from $\mathrm{RC}$ elements tested in the lab and in existing buildings, it would provide invaluable information for the development of RC models. This would inevitably lead to material use reductions in design and more accurate assessments moving forward.

Unfortunately, the manner that reinforcement strains are commonly being measured has remained stagnant for decades (Mains, 1951; Masukawa, 2012). Foil strain gauges are almost always used to measure strain in the lab, which are a discrete technology that only measure strains at a single location. When distributed reinforcement strain measurements are required, which is crucial data for RC cracking and tension stiffening models (Kaklauskas, 2017; Scott and Gill, 1987), it is not uncommon for tens of strain gauges to be bonded sequentially on a single bar (Masukawa, 2012; Scott and Gill, 1987). This is not cost effective and is labour intensive which limits the available number of experiments, thus there is a need for a more practical method of acquiring distributed reinforcement strain measurements.

Current monitoring technologies for measuring in-situ behaviour of RC elements possess several limitations as well. For instance, most available deflection and crack width monitoring devices are also discrete technologies. To measure deflections, reference points are often required, and to measure crack widths, the crack location must be known. Though digital image correlation (DIC) can overcome some of these limitations, it requires a direct line of sight between the camera 
and the structural element. This creates several obstacles when attempting to monitor behaviour on site. If one sensing technology could monitor distributed RC deflections and crack widths simultaneously in a practical manner, while avoiding the limitations mentioned, it could then be a useful tool for monitoring and understanding the response of $\mathrm{RC}$ elements in the field.

The following paper highlights the results of a research program aimed at using Rayleigh backscatter-based distributed fibre optic sensors (FOS) to address the RC monitoring challenges discussed. Techniques were developed for feasibly monitoring reinforcement strains, $\mathrm{RC}$ bending deflections, and crack widths, which will are all discussed here. Additionally, a field monitoring program demonstrating the unique capabilities of using distributed FOS to monitor the response of RC building elements in situ when subjected to live loading is introduced.

It should be noted that there have been previous attempts by researchers using this technology to measure reinforcement strains (Barrias et al., 2018; Brault et al., 2015), RC deflections (Regier and Hoult, 2014) and crack widths (Regier and Hoult, 2014; Rodriguez et al., 2015). However, the research program that is summarized in this paper is the first instance that a method for accurately measuring distributed reinforcement strains beyond concrete cracking is presented. Furthermore, it is the only work that has evaluated the accuracy of RC deflection and individual crack width measurement methods based on this distributed strain measuring technique.

\section{Experimental program}

\subsection{Lab investigations}

The research program that is discussed in this paper included an experimental study consisting of $18 \mathrm{RC}$ beam specimens (full details on the lab investigation are found in Brault (2018), Brault and Hoult (2019a), and Brault and Hoult (2019b). Only the results from three of these specimens will be discussed here: Beam 1, Beam 2, and Beam 3. The cross section of each beam is shown in Figure 1. Beam 1 had a loaded span of $1 \mathrm{~m}$ and Beams 2 and 3 had loaded spans of $2 \mathrm{~m}$. The stirrups were spaced at $125 \mathrm{~mm}$ in Beam 1 and $150 \mathrm{~mm}$ in Beams 2 and 3

Each Beam was loaded until failure in three-point bending using a hydraulic actuator as shown in Figure 1(b). For each beam, linear potentiometers (LPs) were used to measure beam deflections at the supports, the quarter spans, and at mid-span. The LPs at the supports were used to cancel out any support movement. Digital image correlation (DIC) was used to measure crack widths in the central portion of each beam using Canon T3i cameras and a software package called GeoPIV originally developed by White et al. (2003).

Commercially available nylon coated single mode fibre optic cables were used in this investigation $(\sim 0.15 / \mathrm{m})$ as the distributed FOS. As seen in Figure 1(b) and Figure 2(a), FOS were bonded to the external surface of each beam at two separate heights: $10 \mathrm{~mm}$ below the top extreme compression fibre and at the height of the bottom (tensile) reinforcement. FOS were also installed longitudinally along the bottom (tensile) reinforcement within each beam. Figure 2(b) shows the FOS bonded to the bottom reinforcement, and how the fibre was coated in a thin layer of silicone. Both the external and internal FOS passes extended along the entire loaded span of each beam (details regarding the fibre installation process are found in Brault and Hoult (2019a) for the external FOS and Brault and Hoult (2019b) for the FOS on the reinforcement.

Figure 1 Lab specimens and test setup: (a) beam cross sections, and (b) three-point bending test setup where $L$ is the loaded span (all units in $\mathrm{mm}$ ).

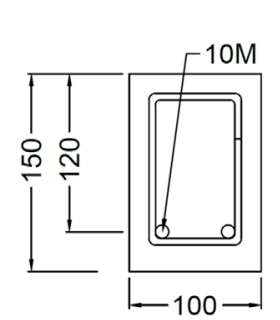

Stirrups diameter $=5 \mathrm{~mm}$ Beam 1

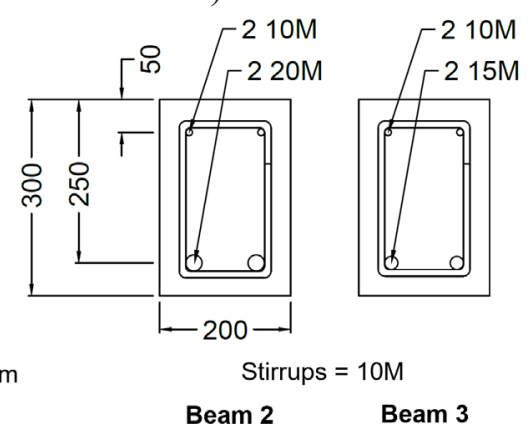

(a)

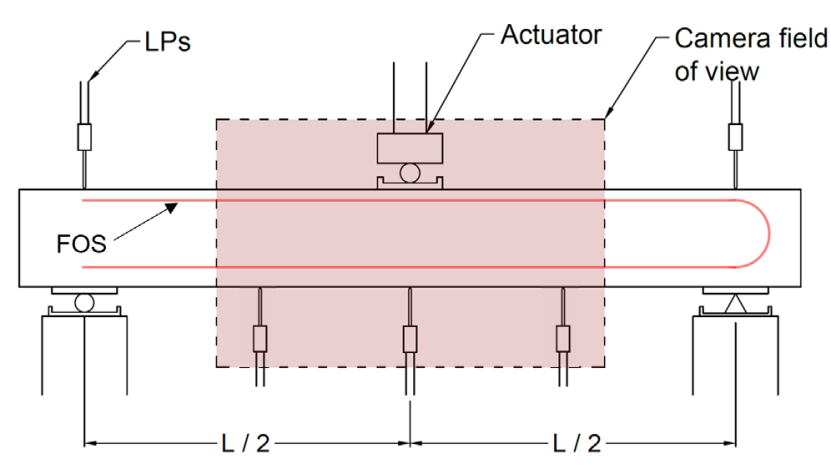

(b) 
Figure 2 Schematic of FOS instrumentation: (a) external concrete surface installation, and (b) bottom (tensile) reinforcement installation.

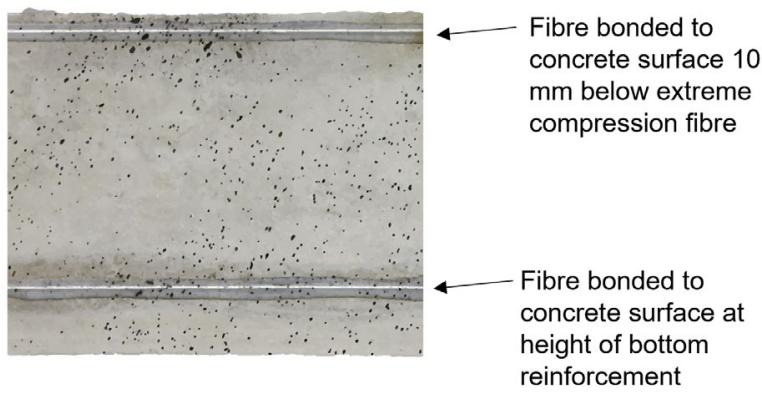

(a)

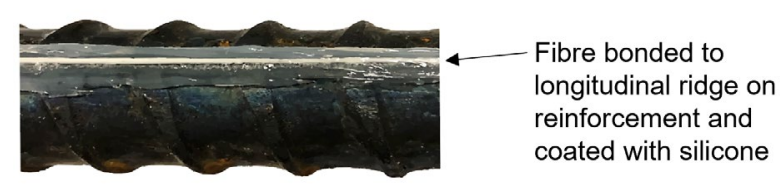

(b)

To acquire data from the FOS, the OBR 4600 analysing unit from Luna Technologies was used. This system is capable of performing distributed strain measurements along a fibre optic cable with a strain resolution of $\sim 1 \mu \varepsilon$ in the sensing core when a sensor spacing and gauge length of $10 \mathrm{~mm}$ is used (Luna Technologies, 2018). All FOS results from the lab that are discussed in this paper used a sensor spacing and gauge length of $10 \mathrm{~mm}$. The maximum fibre length the system can perform strain measurements along is $70 \mathrm{~m}$. Lastly, it is important to note that this fibre optic analyser is a static measurement tool. Thus, in order to perform accurate strain measurements it was necessary to perform each test in a series of increasing load stages (for example: $5 \mathrm{kN}, 10 \mathrm{kN}$, etc.) with FOS measurements being taken once the loading was halted at each stage.

\subsection{Field monitoring}

This research program investigated the instrumentation and testing of three separate beam elements within a 5 storey RC building in Ottawa, Canada. However, only the results from one of these beams will be discussed in this paper, which will be referred to as the 'Large Beam' beyond this point. Full details of the field monitoring portion of this research program are found in Brault and Hoult (2019c). A schematic of the Large Beam is shown in Figure 3. It has a clear span of $15.8 \mathrm{~m}$ and is supported by two steel columns. Cross section views of the Large Beam are shown in Figure 3, however, the beam's reinforcement details are not shown in this figure. FOS were bonded to the external surface of the beam at two separate heights along the beam's span in a similar manner to the external FOS bonded to the lab specimens previously described. There is a beam framing into the Large Beam and there is a pipe installed on the surface of the Large Beam as indicated in Figure 3, which both provided obstacles for the FOS installation. This led to gaps in the FOS data that will be discussed later. Furthermore, FOS were not able to be installed all the way to the centre of the south column as initially intended. This was due to physical obstacles being present on the construction site. The FOS were installed 3 months following the concrete placement when all shores, formwork, and re-shores had been removed.

Figure 3 Large beam schematic with FOS configuration depicted.
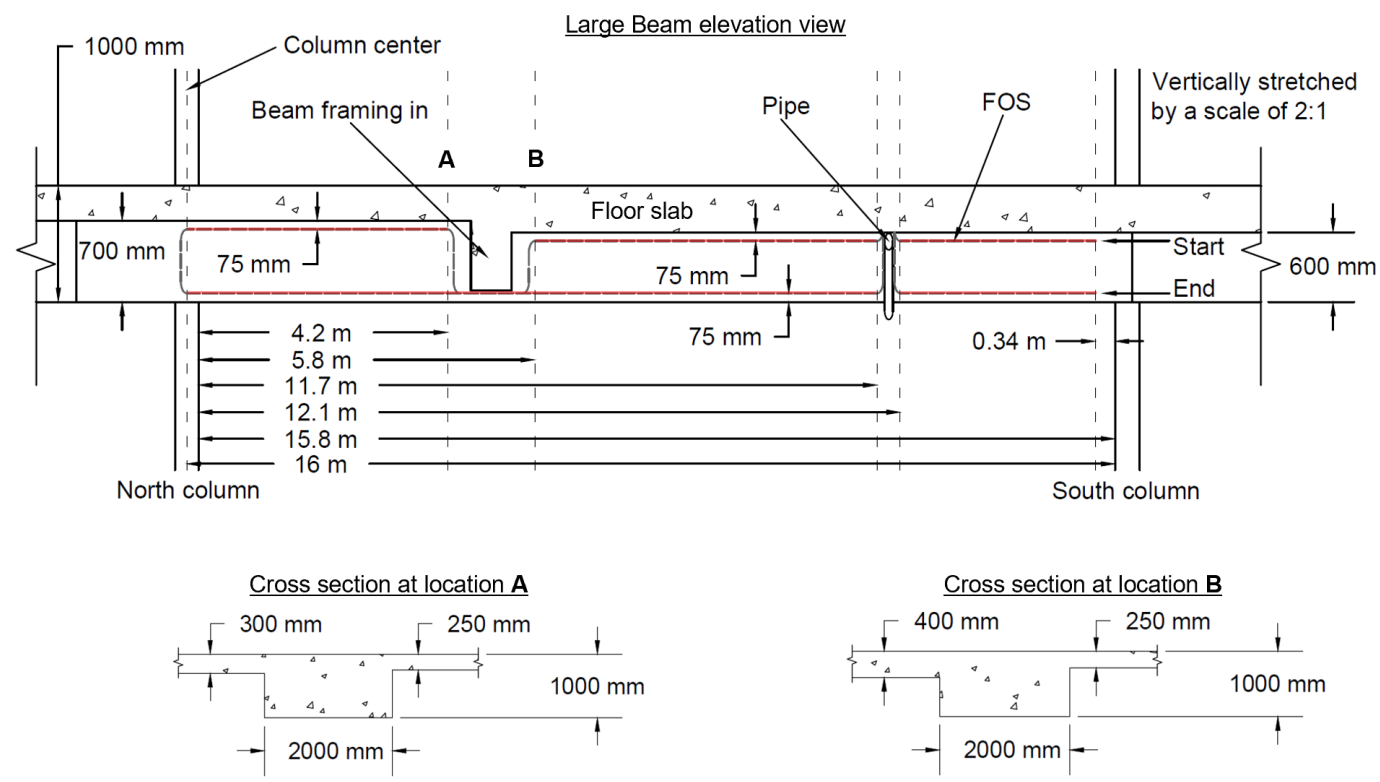
A live load test was performed on the Large Beam to assess the ability of the FOS to monitor the element's response to external loading. As mentioned, the FOS were installed following the removal of all shoring so it could not be used to measure the beam's response to self-weight. The Large Beam was loaded by driving six scissor lifts onto the beam. The scissor lifts were centred along the longitudinal axis of the beam and were oriented parallel to this axis. The lifts each weighed between $11.5 \mathrm{kN}$ and $15.9 \mathrm{kN}$, and each lift's weight was assumed to be evenly split between its two axles. The magnitude and location of every axle load applied to the Large Beam can be seen in Figure 4. Once all six scissor lifts had been driven into place, FOS strain measurements were acquired. Again, the OBR 4600 analysing unit was used to acquire these measurements. However, a $20 \mathrm{~mm}$ sensor spacing and gauge length was used here instead of the $10 \mathrm{~mm}$ setting used for the lab experiments (see Brault and Hoult (2019c) for details)). To measure midspan deflection of the Large Beam, LP measurements were acquired at $1 \mathrm{HZ}$ throughout the entirety of the test. The test was conducted during the building's construction and took under an hour to complete. The brief duration of the test and the fact that the construction site was enclosed eliminated the need for temperature compensation of the FOS measurements, as the temperature change over this time was negligible.

Figure 4 Live loading configuration on Large Beam.

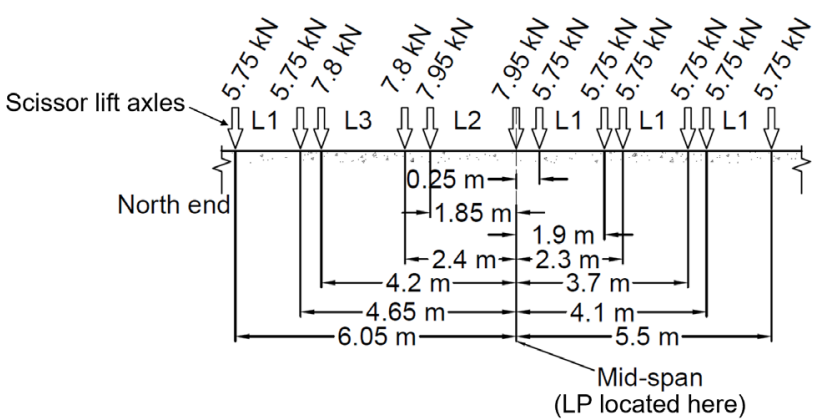

\section{Lab Results}

\subsection{Concrete surface strains}

Figure 5 shows concrete surface strain results measured using the FOS from specimen Beam 1 with an applied of $18 \mathrm{kN}$. This is approximately $50 \%$ of the beam's ultimate load. As expected in positive bending, the top fibre measures compressive strains and the bottom fibre measures tensile strains. The positive 'spikes' in the bottom fibre measurements represent locations in the fibre optic cable that cross cracks in the concrete surface. Therefore, one is able to determine the location of each crack using FOS, meaning that the crack spacing can also be deduced. Brault and Hoult (2019a) showed that an integration process can be applied to these positive strain spikes to estimate the widths of each crack that the fibres cross. For instance, with this technique, the mid-span crack in Figure 5 is estimated to be $0.08 \mathrm{~mm}$ at the height of the bottom fibre optic cable (which is at the height of the bottom reinforcement).
These crack width estimates were evaluated against DIC crack width measurements by Brault and Hoult (2019a), and it was found that their accuracy reduces when crack widths exceed $0.15 \mathrm{~mm}$. This will be discussed further later.

Figure 5 FOS concrete surface strain measurements from Beam 1 with an applied load of $18 \mathrm{kN}$ ( $\sim 50 \%$ of ultimate load).

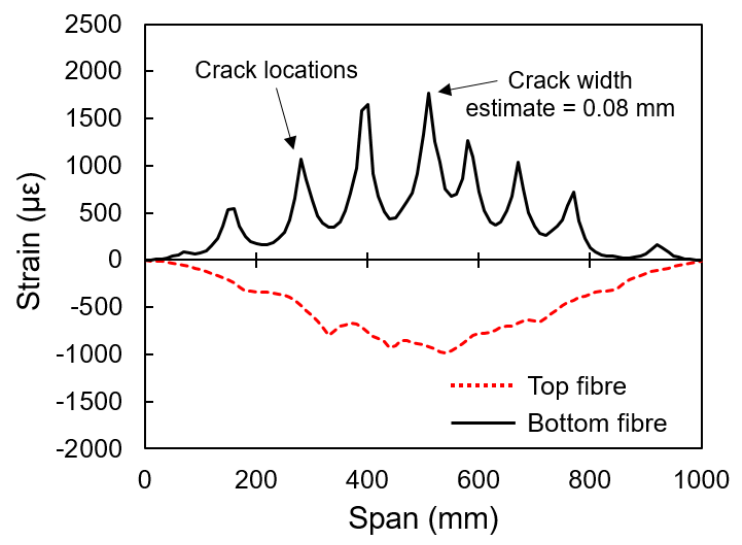

As seen in Figure 5, longitudinal surface strains at two separate heights along Beam 1's entire loaded span are measured. Since the vertical distance between the two fibre passes is known (110 $\mathrm{mm}$ in this case), the beam's curvature can be determined every $10 \mathrm{~mm}$ along the span (this is the sensor spacing used in this test). Using Euler-Bernoulli beam theory, deflections can then be determined by a process of double integration. However, two known boundary conditions must be available to complete this process. In the case of Beam 1 (and all lab tests in this program), both supports were used as boundary conditions with a bending deflection value of $0 \mathrm{~mm}$. It should be noted that the strain results in Figure 5 were not filtered in anyway prior to the process of double integration. Deflection results determined from the external FOS strain measurements are presented in Figure 6 for Beam 1 at a load of $18 \mathrm{kN}$.

Figure 6 Comparison between FOS deflection measurements and LP measurements for Beam 1 with an applied load of $18 \mathrm{kN}$.

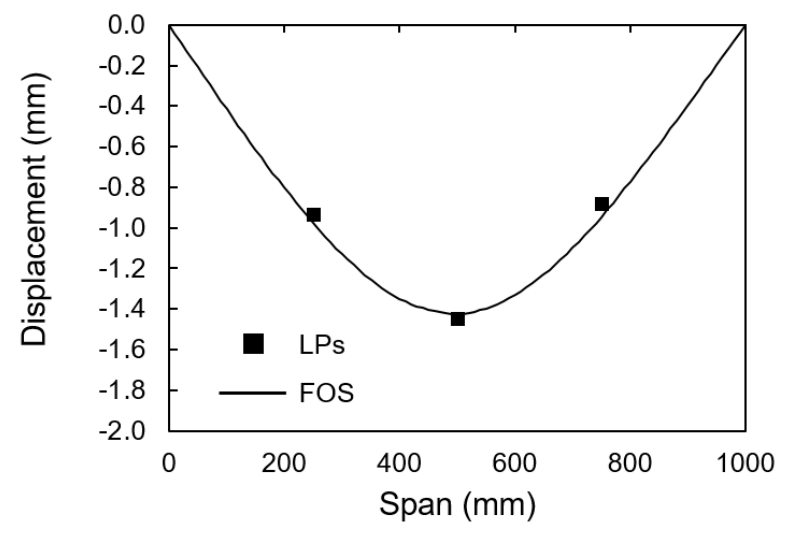


Figure 6 shows that the FOS were able to capture the full deflected shape of Beam 1. The FOS deflection results are in good agreement with the LP measurements at both quarterspans and at mid-span (the maximum difference is $0.06 \mathrm{~mm}$ ). As seen in Figure 1(b), both the top and bottom fibre passes are comprised of a single fibre optic cable. Thus, Figure 6 shows that the full deflected shape (containing 100 sequential deflection points in this case as a result of the $10 \mathrm{~mm}$ sensor spacing) can be measured using a single fibre optic cable. Brault and Hoult (2019a) found that once the fibre used to measure deflections is required to cross cracks of a certain width, the accuracy of the FOS deflection measurements begins to reduce significantly. This is discussed later in more detail.

In Brault and Hoult (2019a), crack width estimates performed using FOS measurements are evaluated against DIC crack width measurements from 11 different RC beam specimens. These results are presented in Figure 7, with good correlation between measurement technologies being represented by the $1: 1$ line that is plotted. It is visually evident in Figure 7 that the accuracy of the FOS crack width estimates decreases for crack widths larger than $0.15 \mathrm{~mm}$. In fact, the average measurement difference up until $0.15 \mathrm{~mm}$ is only $0.007 \mathrm{~mm}$ while it increases to 0.033 ( $\sim 5$ times higher) beyond that point. This decrease in accuracy was found to be caused by severe strain gradients in the FOS at cracks, leading to the occurrence of unreliable strain readings at these locations (further details in Brault and Hoult (2019a)). Overall, the FOS were determined to estimate crack widths accurately up until widths of $0.15 \mathrm{~mm}$ and can be used to locate all crack locations that the fibre optic passes. Beyond $0.15 \mathrm{~mm}$ the FOS may provide a reasonable crack width estimate up to $0.3 \mathrm{~mm}$, and if more accurate measurements are required then conventional crack gauges can be installed where the FOS results suggest the most critical cracks to be located.

Figure 7 Evaluation of FOS crack width estimates compared to DIC crack width measurements from 11 different RC beam specimens.

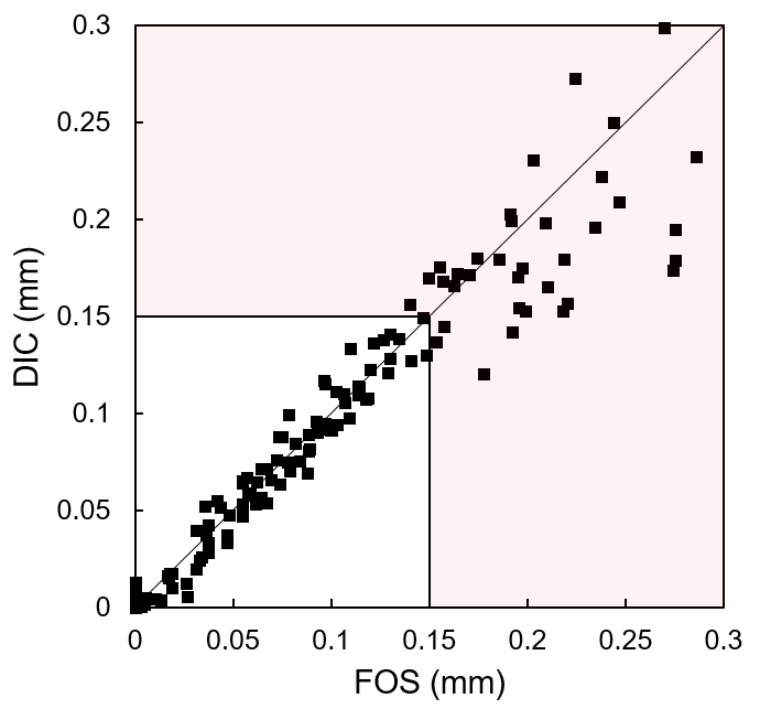

Brault and Hoult (2019a) also evaluated deflection results determined using the external FOS strain measurements from the same 11 RC beam specimens used in Figure 7. A comparison between LP and FOS deflection measurements at each beam's mid-span is shown in Figure 8. Again, agreement between measurement technologies is represented by the $1: 1$ line that is plotted. Figure 8 shows that the FOS provide accurate deflection measurements until the crack widths are $0.15 \mathrm{~mm}$ or greater. Once either of the fibre passes used to perform the deflection measurements cross a crack with a width greater than $0.15 \mathrm{~mm}$, the accuracy of the deflection measurements starts to decrease. This is not surprising, as it was previously discussed that unreliable strain readings start to occur in the fibre optic cables once cracks with widths larger than $0.15 \mathrm{~mm}$ are crossed by the fibres themselves. Since accurate strain at each point along the FOS is required to perform the integration needed to determine bending deflections, this also leads to inaccurate FOS deflection results. Fortunately, it is possible to avoid this issue by installing at least two fibre optic passes at beam depths that are not expected to experience concrete cracking. This strategy is further discussed in Brault and Hoult (2019a).

Figure 8 Evaluation of FOS deflection measurements compared to LP measurements at mid-span for $11 \mathrm{RC}$ beam specimens.

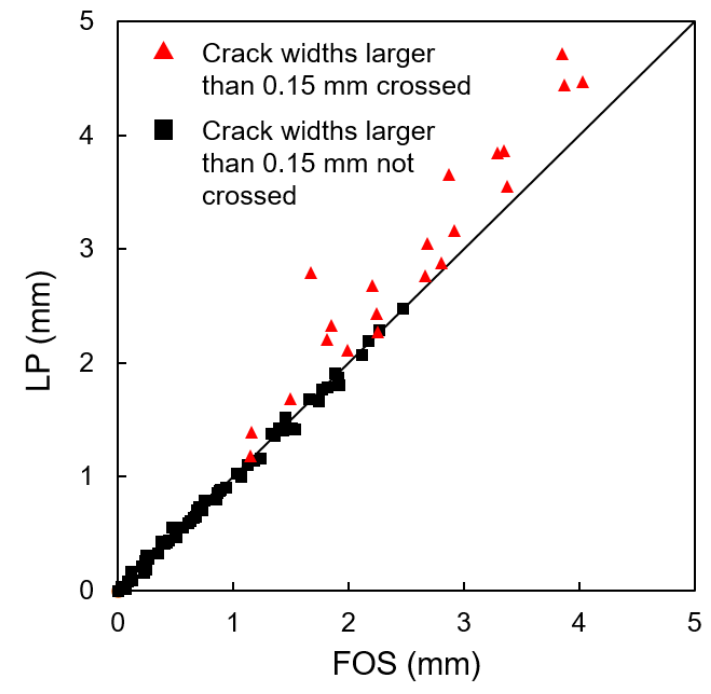

Overall, this section highlights that several crack widths and the deflected shape of an RC beam can be accurately and feasibly measured using a single fibre optic cable bonded to the beam's surface. However, it is important to consider the limitations discussed before implementation.

\subsection{Reinforcement strains}

Measuring distributed strain profiles provides crucial data for developing cracking, tension stiffening, and assessment models for RC structures. However, the cost and labour that are required to achieve this using current monitoring techniques make it too cumbersome to readily perform. For example, a test performed by Masukawa (2012) required the 
installation of 62 strain gauges on a $1 \mathrm{~m}$ long $\mathrm{RC}$ specimen to measure distributed strains. Distributed FOS provide a far more practical option for gathering this data, as is presented next.

Figure 9 displays reinforcement strains measured with FOS for specimen Beam 3 at six different load stages. Beam 3 had a cracking load of $17 \mathrm{kN}$, a yielding load of $95 \mathrm{kN}$, and an ultimate load of $108 \mathrm{kN}$. The strains shown were measured on one of the specimen's bottom reinforcement bars, which was a $15 \mathrm{M}$ deformed bar. In the load stages beyond concrete cracking $(17 \mathrm{kN})$, peaks and valleys are evident in the data. The peaks are a strain increase in the reinforcement at cracks in the concrete, and the valleys are a decrease in strain between cracks. This behaviour makes sense as the reinforcement is expected to carry the tensile stress across a crack, while between cracks some of the force is transferred into the surrounding concrete through bond. This data also has the added value of being able to capture the strain in the reinforcement at every crack location without having prior knowledge as to where each crack will occur.

The accuracy of this data is validated both at and between cracks using strain gauges and theoretical predictions in another paper (Brault and Hoult, 2019b). The data is also visually comparable to the behaviour observed by Masukawa (2012) when measuring distributed strains with 62 side-by-side strain gauges. The strain profiles seen in Figure 9 are from a single fibre optic cable and encompass 200 side-by-side strain measurements, presenting a promising technique for acquiring these results moving forward.

Figure 9 FOS strains measured on a bottom reinforcing bar $(15 \mathrm{M})$ in Beam 3 at increasing load stages.

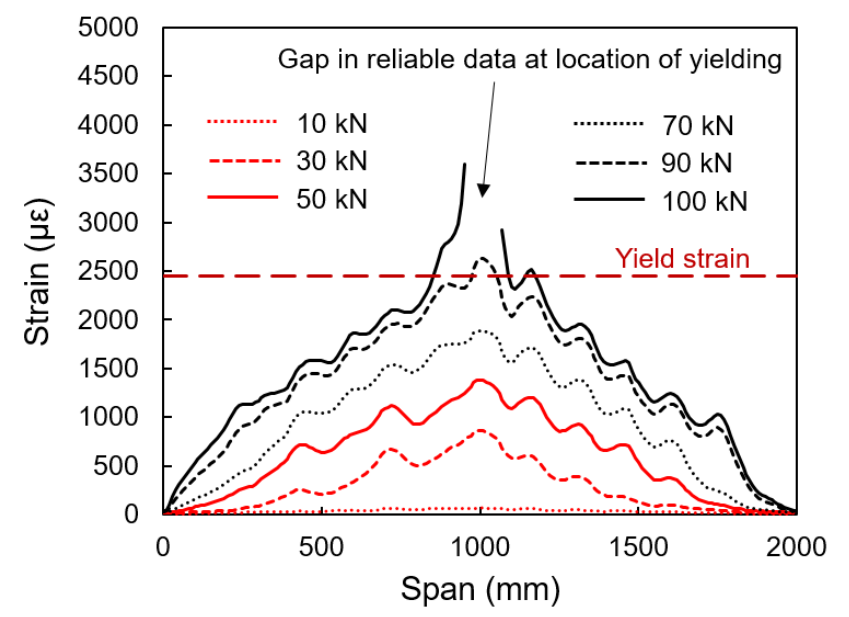

It is evident in Figure 9 that there is a gap in reliable data at mid-span at the $100 \mathrm{kN}$ load stage. This coincides with the location at which the reinforcement started to yield, and at a load stage higher than the yield load $(95 \mathrm{kN})$. The concrete crack where the reinforcement starts yielding begins to grow significantly. This crack growth likely leads to a shearing of the fibre optic cable, which then leads to un-reliable strain readings at this point in the fibre. It also leads to rupturing of the fibre optic cable in some cases. Brault and Hoult (2019b) found that measuring reinforcement strains beyond yielding at the location of yielding is usually not possible using this technique, which is an important limitation to consider moving forward.

Figure 9 also shows that the yielding strain is exceeded in the $90 \mathrm{kN}$ load stage strain profile. In fact, it is exceeded by roughly $200 \mu \varepsilon$. An offset of this magnitude is something that can be caused by shrinkage (Bischoff, 2001; Davis et al., 2017), which imposes compressive strains on the reinforcement prior to testing.

Figure 10 presents a comparison between distributed reinforcement strains and external concrete surface strains at the height of the bottom reinforcement. The data in Figure 10 is from specimen Beam 2 at a load of $40 \mathrm{kN}(\sim 30 \%$ of the ultimate load). The crack locations from both sets of data are in agreement. As expected, the strains in the fibre optic cable bonded to the surface of the concrete experiences much greater strain magnitudes at cracks than measured in the reinforcement. Moreover, the strain 'valleys' are deeper for the external fibre measurements. This again makes sense as these measurements should demonstrate longitudinal deformation compatibility from one end of the beam to the other. In this particular case, there is a $1 \%$ difference in the summation of the strains between supports, which may be caused by the fibre pass heights not being precisely the same. Figure 10 introduces a potential opportunity for external fibre measurements to be used as a means of determining internal stress states. However, this has not yet been investigated further and will be the topic of future research.

Figure 10 Comparison between bottom reinforcement strains and external concrete strains measured at the height of the bottom reinforcement (Beam 2 at a load of $40 \mathrm{kN})$.

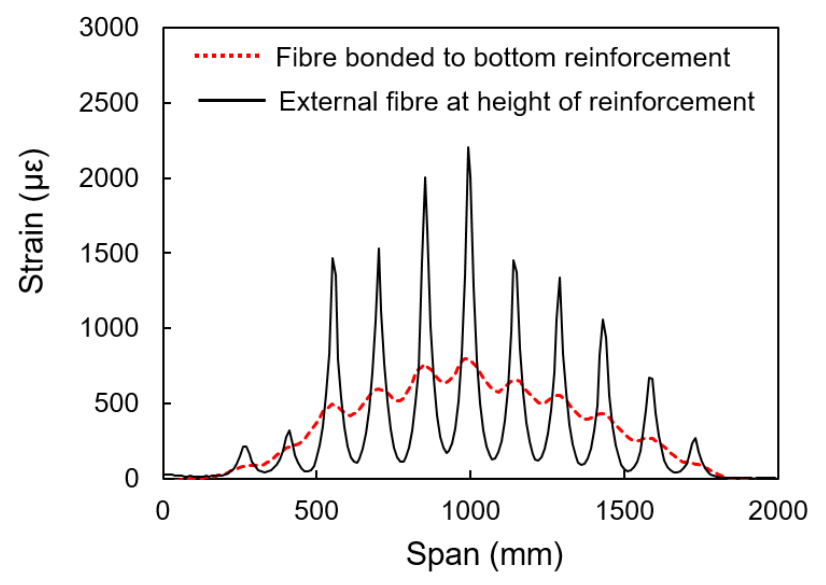

\section{Field results}

The Large Beam's response to the live loading shown in Figure 4 is presented in Figure 11. The shear force diagram, bending moment diagram, FOS strain measurements, beam slopes, and full deflected shape are shown. 
Figure 11 Large Beam FOS results from the live load test.
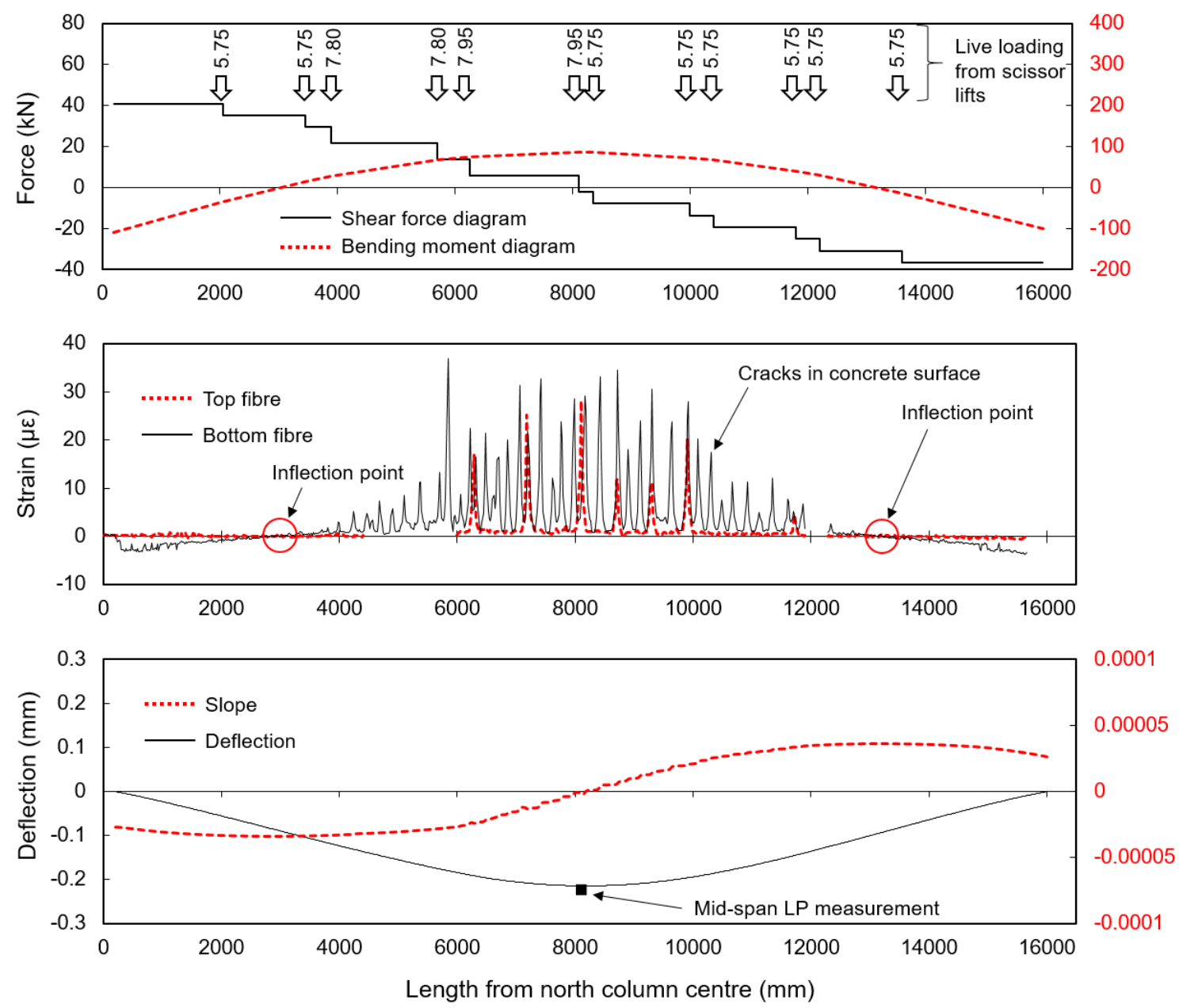

In Figure 11, the bottom fibre measures larger tensile strains than the top fibre near mid-span, indicating positive bending in this location as expected. One can also see the presence of several cracks in the concrete, some of which are seen to extend up to the height of the top fibre. This is evident by spikes in the top fibre strain measurements. The FOS crack width measurement techniques described by Brault and Hoult (2019a) could be used here to determine how much each crack has opened due to the scissor lift loading. It should be noted that the actual crack widths cannot be determined here as the FOS was installed when the element was already subjected to its self-weight and thus already cracked.

The top and bottom fibre measurements are seen to intersect at two distinct points in Figure 11. These points are inflection points, and represent locations of zero bending moment. With the known loading configuration, and the two known bending moments $(0 \mathrm{kNm}$ at $3000 \mathrm{~mm}$ and $13240 \mathrm{~mm})$, a unique solution for both the shear force and bending moment diagrams caused by the live loading can be solved. These are both shown in Figure 11. In this manner, one can understand the moment distribution along the Large Beam's span, highlighting the negative bending at each support. The amount of moment that the Large Beam transfers into the surrounding elements at each support can also be directly determined. For example, there is $110 \mathrm{kNm}$ transferred at the north column and $100 \mathrm{kNm}$ transferred at the south column from the scissor lift loading.

The gaps in the FOS strain data are due to physical obstacles on site as shown in Figure 3 (a beam framing in and a pipe). As the deflection integration process requires curvature values at every point along the elements length to complete, linear strain interpolation was performed across these data gaps. Using the same integration process discussed previously, the full deflected shape of the Large Beam can be determined from the FOS results if two boundary conditions are assumed. Again, the supports were taken to have bending deflection values of 0 $\mathrm{mm}$. The deflected shape of the Large Beam is shown in Figure 11 , which displays some negative curvature near both supports as expected (consistent with the FOS strains and the bending moment diagram). The FOS deflection measurement at midspan is in good agreement with the LP measurement as well, though there is a slight error $(0.009 \mathrm{~mm})$.

Ultimately, Figure 11 demonstrates the extensive amount of information that is achievable from installing a single fibre 
optic cable at two separate heights on an RC element in situ (the full field monitoring program is detailed in Brault and Hoult (2019c)).

\section{Conclusions}

This paper presented an overview of a research program that was aimed at determining the capabilities of monitoring and assessing RC behaviour with Rayleigh-based distributed FOS In all cases discussed here, the loading was applied in a single direction. Therefore, future work is required to comment on the accuracy of this system under cyclic loading conditions. The following key conclusions can be made from this study:

- FOS bonded to the surface of concrete elements can be used to accurately measure crack widths and distributed deflections until the fibre optic cable crosses cracks larger than $0.15 \mathrm{~mm}$ in width.

- FOS bonded to steel reinforcement embedded in $\mathrm{RC}$ elements can measure distributed strains in a manner that is far more practical than is possible with strain gauges. However, accurate measurements are usually not achievable at the location of yielding.

- Pairing reinforcement strain measurements with measurements from the concrete surface presents an interesting opportunity for determining internal steel stress states from externally bonded FOS. Further research is required in this area.

- FOS installed on RC elements in the field can provide detailed insight into support conditions, cracking behaviour, load distribution, and deflection.

\section{Acknowledgements}

The authors would like to thank Queen's University, the Natural Sciences and Engineering Research Council of Canada (NSERC), the Ontario Ministry of Research and Innovation, and the Canadian Foundation for Innovation for financially supporting this work. The authors also extend their gratitude to Rebecca Garratt, Adam Hoag, Sara Nurmi, Kyle Van Der Kooi, Jon Remacka, Jiachen Zhang, Jack Poldon, Paul Thrasher, and Neil Porter from Queen's University. Lastly, sincere thanks is owed to Tom Greenough and Ian Trudeau of Entuitive Corp and Bryce Howchin and Tanner Blom of PCL.

\section{References}

Andrew RM (2018) Global $\mathrm{CO}_{2}$ emissions from cement production. Earth Systems Science Data 10: 195-217.

Barrias A et al. (2018) Embedded distributed optical fibre sensors in reinforced concrete structures - a case study. Sensors 18(4): 1-22.

Bischoff PH (2001) Effects of shrinkage, tension stiffening, and cracking in reinforced concrete. Canadian Journal of Civil Engineering 28(3): 363-374.

Brault A et al. (2015) Development of a relationship between external measurements and reinforcement stress. Proc. SPIE 943, Sensors and Smart Structures Technologies for Civil, Mechanical, and Aerospace Systems, 2015, San Diego, CA, U
Brault A (2018) Novel sensors for improving reinforced concrete design and assessment. PhD dissertation, Department of Civil Engineering, Queen's University Kingston, ON, Canada.

Brault A and Hoult NA (2019a) Monitoring RC serviceability performance using fiber optic sensors. ACI Structural Journal 116(1): 57-70.

Brault A and Hoult NA (2019b) Distributed reinforcement strains: measurement and application. ACI Structural Journal, in press.

Brault A and Hoult NA (2019c) Monitoring of beams in an RC building during a load test. ASCE Journal of Performance of Constructed Facilities 33(1): 04018096 1-11.

Davis M et al. (2017) Distributed sensing for shrinkage and tension stiffening measurement. ACI Structural Journal, 114(3): 753-764

Kaklauskas G (2017) Crack model for RC members based on compatibility of stress-transfer and mean-strain approaches. ASCE Journal of Structural Engineering 143(9): 04017105 112

Liew A (2017) Design, fabrication and testing of a prototype, thin-vaulted, unreinforced concrete floor. Engineering Structures 137: 323-335.

Luna Technologies (2018) OBR 4600 optical backscatter reflectometer data sheet. https://lunainc.com/wpcontent/uploads/2012/11/OBR-4600-Data-Sheet-9-Mar-

2018.pdf (accessed 14/01/2019)

Mains RM (1951) Measurement of the distribution of tensile and bond stresses along reinforcing bars. ACI Journal Proceedings 48(11): 225-252.

Masukawa J (2012) Degradation of shear performance of beams due to bond deterioration and longitudinal bar cutoffs. PhD dissertation, Department of Civil Engineering, University of Toronto, Toronto, ON, Canada.

Orr JJ (2012) Flexible formwork for concrete structures. PhD dissertation, Department of Architecture and Civil Engineering, University of Bath, Bath, UK.

Regier R and Hoult NA (2014) Distributed strain behaviour of a reinforced concrete bridge: case study. ASCE Journal of Bridge Engineering 19(12): 05014007 1-9.

Rodriguez G et al. (2015) Cracking assessment in concrete structures by distributed optical fibre. Smart Materials and Structures, 24(3): 035005 1-11.

Scott RH and Gill PAT (1987) Short-term distribution of strain and bond stress along tension reinforcement. The Structural Engineer 65(2): 39-43.

United Nations Department of Economics and Social Affairs (2018) 2018 revisions of world urbanisation prospects. https://www.un.org/development/desa/publications/2018revision-of-world-urbanization-prospects.html (accessed 14/01/2019)

White DJ et al. (2003) Soil deformation measurement using particle image velocimetry (PIV) and photogrammetry. Geotechnique 53(7): 619-632. 